\title{
Application of an optimized pharyngeal swab-assisted device in pharyngeal sampling for COVID-19 patients
}

\author{
Jie Jia ${ }^{1}$, Sun-Ting Fang ${ }^{1}$, Xiao-Bo Feng ${ }^{1}$, Wei Tong ${ }^{1}$, Hong-Tao Tian ${ }^{1}$, Jing Tang ${ }^{2} \wedge$ \\ ${ }^{1}$ Department of Orthopedics, Union Hospital, Tongji Medical College, Huazhong University of Science and Technology, Wuhan, China; ${ }^{2}$ Cancer \\ Center, Union Hospital, Tongii Medical College, Huazhong University of Science and Technology, Wuhan, China \\ Contributions: (I) Conception and design: J Tang, J Jia; (II) Administrative support: HT Tian; (III) Provision of study materials or patients: J Jia, XB \\ Feng; (IV) Collection and assembly of data: ST Fang; (V) Data analysis and interpretation: J Tang, W Tong, XB Feng; (VI) Manuscript writing: All \\ authors; (VII) Final approval of manuscript: All authors. \\ Correspondence to: Jing Tang, MD, PhD. Cancer Center, Union Hospital, Tongji Medical College, Huazhong University of Science and Technology, \\ Wuhan 430022, China. Email: michelleyiyi@hotmail.com; Hong-Tao Tian, MD. Department of Orthopedics, Union Hospital, Tongji Medical \\ College, Huazhong University of Science and Technology, Wuhan 430022, China. Email: tianhongtao@vip.163.com.
}

Background: Coronavirus disease 2019 (COVID-19), which is caused by severe acute respiratory syndrome coronavirus 2 (SARS-CoV-2), has triggered a global pandemic. Healthcare workers are placed at an elevated risk of nosocomial cross-infection from clinical exposure. One diagnostic criterion for COVID-19 is a positive result from a real-time reverse transcriptase-polymerase chain reaction (qRT-PCR) assay of pharyngeal swab specimens, which has been a routine procedure for healthcare workers during the outbreak. In the context of a global shortage of personal protective equipment (PPE), we aimed to lower the probability of clinical cross-infection without impacting the results of pharynx sampling through an optimized pharyngeal swab assisted device (OPAD).

Methods: To evaluate the efficacy and feasibility of an OPAD for the detection of SARS-CoV-2, 22 confirmed COVID-19 cases were enrolled in our self-controlled study. The results of two pharyngeal sampling qRT-PCR tests using the OPAD or the traditional method were recorded each. Clinical data including baseline characteristics, laboratory tests, and computed tomography (CT) results were also collected. The procedure duration and levels of pharynx exposure with the OPAD, and the diagnostic consistency between the OPAD and the traditional method for pharyngeal sampling qRT-PCR, were evaluated individually. Additionally, a questionnaire was designed for healthcare workers who had performed the pharyngeal swab to deepen our understanding of their attitude during their service on the frontline.

Results: In all 44 samplings (22 samples with each method), the qRT-PCR results of 18 pairs $(81.82 \%)$ were consistent, while $3(13.64 \%)$ were single positive with the OPAD. The positive rate was slightly higher with the $\operatorname{OPAD}(54.55 \%, 12 / 22)$ than with the traditional method $(45.45 \%, 10 / 22)$. Using the OPAD, the average procedure duration of sampling was $30 \mathrm{~s}(30 \pm 13 \mathrm{~s})$. Pharynx exposure was excellent in 21 subjects $(95.45 \%, 21 / 22)$, which meant that the operator could acquire the swabs without difficulty.

Conclusions: As the COVID-19 pandemic escalates, our OPAD has identical efficacy compared to the traditional method for pharyngeal swabs, and it can also contribute to protecting the safety of healthcare workers.

Keywords: Coronavirus disease 2019 (COVID-19); severe acute respiratory syndrome coronavirus 2 (SARSCoV-2); pharyngeal swab; optimized pharyngeal swab assisted device (OPAD); nosocomial cross-infection prevention

^ ORCID: 0000-0003-1013-147X. 
Submitted Apr 28, 2020. Accepted for publication Nov 15, 2020.

doi: 10.21037/atm-20-3612

View this article at: http://dx.doi.org/10.21037/atm-20-3612

\section{Introduction}

In early December 2019, pneumonia cases of unknown origin were identified in Wuhan, China. The disease that caused this outbreak has since become known as coronavirus disease 2019 (COVID-19) (1). As of July 20, 2020, the World Health Organization (WHO) had documented 14,263,202 confirmed cases and 602,244 deaths globally (https://www.who.int/).

For healthcare workers, the COVID-19 pandemic has meant an elevated risk of exposure to infection. The early stages of the COVID-19 outbreak are reminiscent of the severe acute respiratory syndrome (SARS) epidemics, during which healthcare workers accounted for 1,707 (21\%) of the 8098 cases confirmed by the WHO. Unfortunately, the SARS-CoV-2 virus has already infected many healthcare workers through nosocomial transmission. In the 422 medical institutions that have provided diagnosis and treatment for COVID-19 patients in China, 3,019 healthcare workers have been infected with SARS-CoV-2 (1,716 confirmed cases), of whom five have died. According to the analysis of 1,688 of these confirmed cases, 1,441 were mild $(85.4 \%)$, and the case fatality rate was lower than that in other populations (2). Additionally, several studies have reported the prevalence of SARS-CoV-2 infection among healthcare workers in other parts of the world $(3,4)$. In 2003 a study reported on healthcare workers with asymptomatic SARS and their potential for disease transmission (5). Therefore, it is likely that stealth-infected healthcare workers might also participate in the nosocomial crossinfection of SARS-CoV-2. Currently, the specific reasons behind the infection of healthcare workers and the failure of protective measures require further investigation.

One diagnostic criterion for COVID-19 is a positive result from a high-throughput sequencing or real-time reverse transcriptase-polymerase chain reaction (RT-PCR) assay of nasal or pharyngeal swab specimens (6). Although the procedure for obtaining a pharyngeal swab is noninvasive, it may elicit a cough from the patient, which can result in droplet transmission. Thus, this procedure should only be performed by qualified staff with proper personal protective equipment (PPE). Due to the relatively high false-negative rate, most patients require repeated testing to obtain a diagnosis or to fulfill the discharge criteria.
Therefore, pharyngeal swab, rather than endotracheal intubation, is becoming a routine procedure, putting healthcare workers at a greater risk of cross-infection.

Moreover, we believe that the false-negative rates are related to underexposure of the pharynx, insufficient light, and healthcare workers flinching during the procedure. Improving the accuracy of pharyngeal swab tests and reducing the occupational exposure of healthcare workers have become unsolved, urgent problems. Here, compared to the traditional pharyngeal swabbing method, we present an optimized pharyngeal swab assisted device (OPAD) that offers several advantages: improved, or at least uncompromised, efficacy; and lower risk for healthcare workers due to less direct exposure.

We present the following article in accordance with the MADR reporting checklist (available at http://dx.doi. org/10.21037/atm-20-3612).

\section{Methods}

\section{Questionnaire}

A brief questionnaire-based survey (detailed in the Appendix 1) was designed and administrated to a sample of healthcare staff working on the frontline of the SARS-CoV-2 outbreak. Among the 82 eligible respondents, 76 (92.68\%) were based in Hubei, including Wuhan, 4 (4.88\%) worked in other provinces of China, and 2 (2.44\%) were based overseas. To be eligible for inclusion, participants needed to have performed the pharyngeal swab procedure at least once. The purpose of this survey was to evaluate pharyngeal swab sampling in clinical practice with respect to the following points: (I) professionalism of the operator; (II) perceived fear of the operator; (III) infection rate of the operator; (IV) perceived level of protection against SARS-CoV-2; and (V) operational effectiveness of the pharyngeal swab. The results of the questionnaire were then collected, checked for completion, and analyzed.

\section{Design and production of the pharynx exposure and observation device}

\section{Pharynx exposure apparatus}

The pharynx exposure unit was developed by the 
SolidWorks software and constructed with an FDM ${ }^{\mathrm{TM}}$ 3D printer (MakerBot Industries, New York, USA) using acrylonitrile butadiene styrene (ABS). The apparatus is composed of three inseparable parts: (I) the main body of the oris speculum, consisting of a $3.5 \mathrm{~cm}$-high fixed retainer, which is intended to be placed between the upper and lower dentition. Patients can rest with an open mouth, while the distractor stabilizes the interincisor distance. (II) The second part is a tongue spatula, which is a $4.5 \mathrm{~cm}$ long downward semi-arc-shaped structure. After the patient bites the retainer, the tongue depressor is designed to simultaneously press and fix the tongue, exposing the pharyngeal wall. (III) The third part is the camera holder, which can hold the camera in place in front of the teeth, enabling the operator to adjust the pitch of the camera so that the pharyngeal wall can be observed completely without impacting the swabbing procedure. The whole oris speculum is designed for singleuse, but it is also reusable after disinfection following the procedure detailed below (Figure 1).

\section{Display unit}

The display unit consists of a camera with a light source, a data transmission line, and an electronic display screen, which wrapped by a disposable transparent package to avoid cross-infection, and the package must be changed after every single use. This display device could be a laryngoscope, a fiberoptic bronchoscope, or other equipment, such as an industrial endoscope. The prototype was installed with an illuminating electronic industrial endoscope with a diameter of $8 \mathrm{~mm}$ (Inskam Company, Shenzhen, China).

\section{Patients}

All 22 patients were enrolled between March 11th and March 25th, 2020, at Wuhan Union Hospital, which was one of the designated infectious hospitals. A self-controlled case series (SCCS) study method was used to evaluate the accuracy of the RT-PCR results obtained from optimized pharyngeal swab samples from patients who had been confirmed to be actively COVID-19 positive according to WHO interim guidance (Figure 2). All patients included in the trial returned at least one positive test result within a week before their recruitment at Wuhan Union Hospital. The exclusion criteria for our study were: (I) maxillofacial deformities or related underlying diseases; (II) an interincisor distance of less than three transverse fingers; (III) dentition defects and edentulous patients; (IV) critical illness; or (V) inability to comply with the pharyngeal swab.
By March 19, 2020, 22 hospitalized patients aged between 31 and 79 years had participated in the study, which was approved by the Tongji Medical College Ethics Committee. Informed consent was given by all participants before their enrollment and participation in any study-related activities.

\section{Study design}

We hypothesized that the present optimized device for pharyngeal swabs could provide a firmly and entirely exposed field of pharyngeal vision, by which the accuracy of sampling would be increased and concurrently, the risk of nosocomial cross-transmission with life-threatening SARS-CoV-2 would be decreased. This clinical study was conducted in accordance with the principles of the Declaration of Helsinki (as revised in 2013) from the International Conference on Harmonization. Using the OPAD and traditional method respectively, the performances of the two adjacent RT-PCR tests for pharyngeal swabs were appraised. Procedure duration time, the Mallampati score (MS) $(7,8)$, and the compliance and tolerance of the patients using the OPAD were recorded. Patient data on laboratory tests, chest computed tomography (CT), and general condition were acquired along with standard medical records. The severity of COVID-19 pneumonia was defined based on the international guidelines for community-acquired pneumonia (9).

\section{RNA Extraction and RT-PCR}

The nucleic acid tests for SARS-Cov-2 were performed according to the previous WHO recommendation. The pharyngeal swab specimens were obtained from all participants (with the OPAD or traditional method). The RNA was extracted and examined by qRT-PCR with SARSCov-2-specific primers and probes based on the detection kit manual. If one of the two targets $(\mathrm{RdRp}+, \mathrm{E}$ or $\mathrm{N}+)$ tested positive by qRT-PCR, the result was considered to be positive.

A Ct value or $\mathrm{Ct}$ of $\geq 40$ was considered negative, and a $\mathrm{Ct}$ value of $<37$ was deemed positive. A Ct value of 37-40 was considered indeterminate, and a repeat test was required to gain clarity of the result.

\section{Chest CT}

The typical and atypical chest CT findings of all 22 


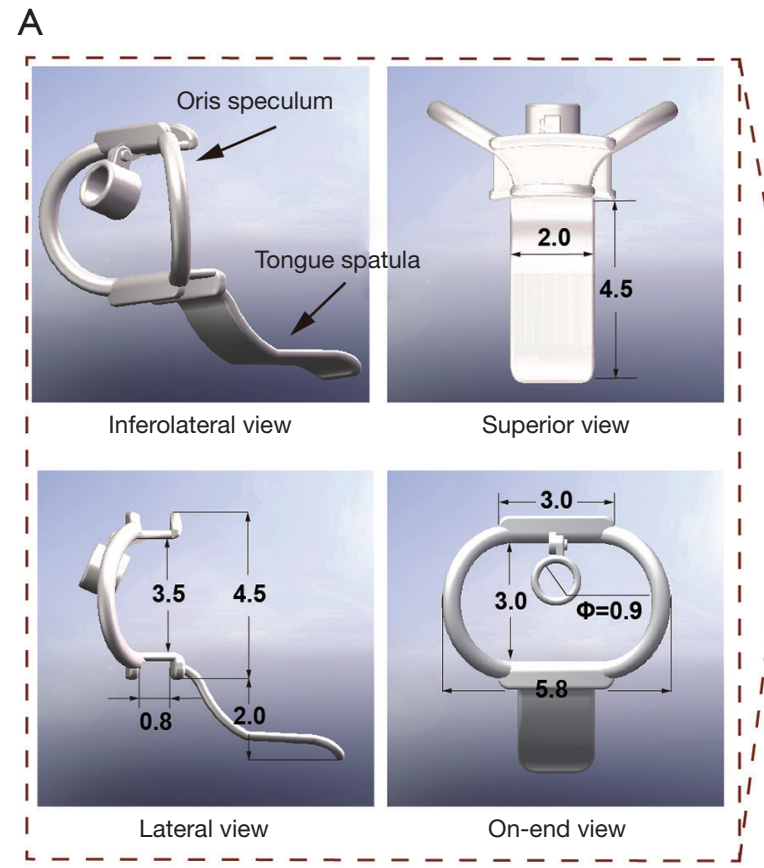

B

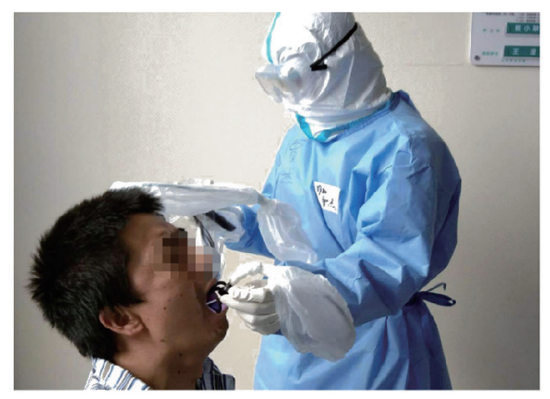

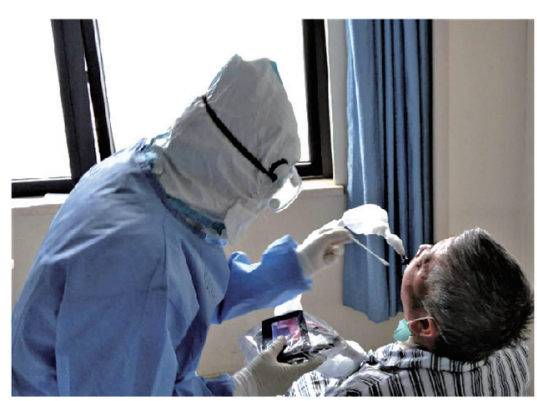

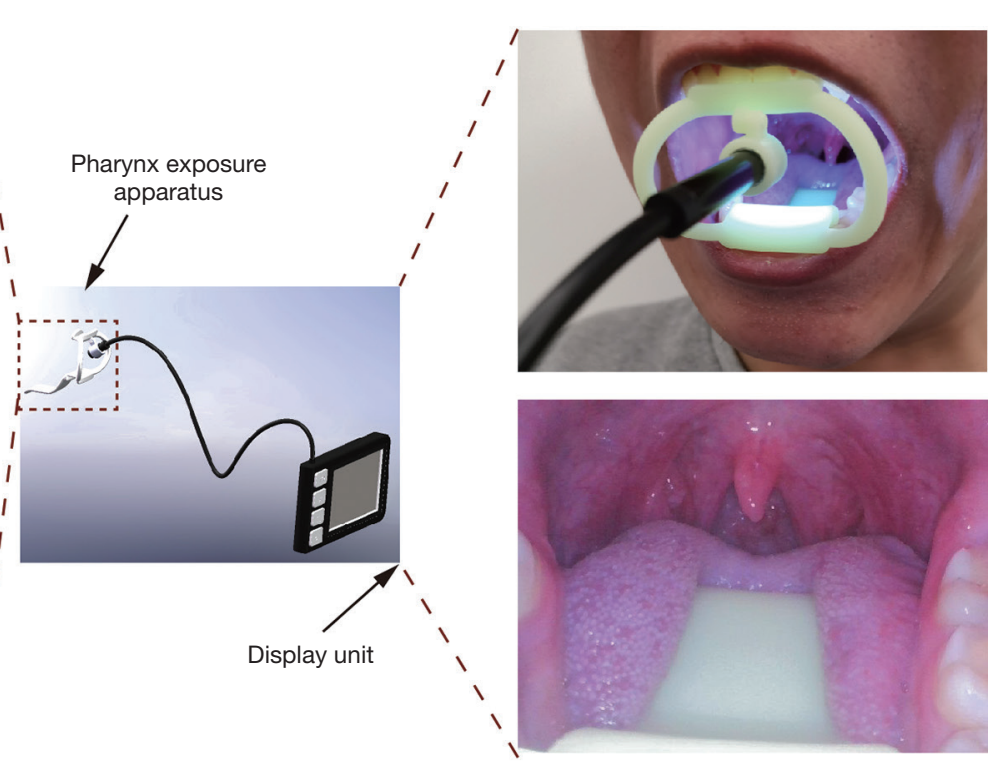

C

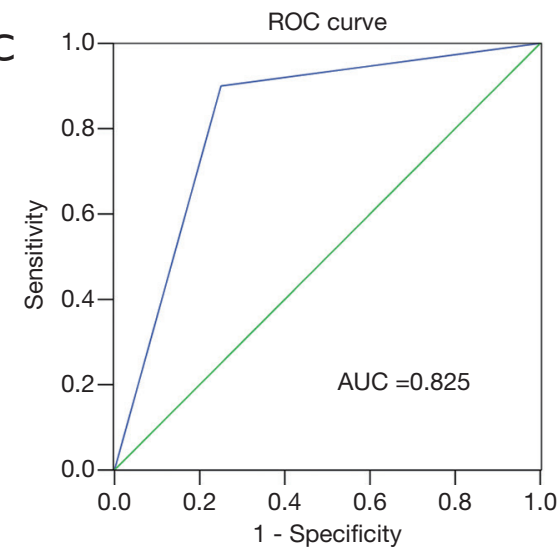

Figure 1 Design and utilization of the OPAD. (A) Left and middle panels: virtual rendering of the OPAD, demonstrating the bounded design parameters of the device; Right panel: the final 3D-printed OPAD used to perform a pharyngeal swab (upper); Grade 1 (MS) exposed the pharyngeal wall and tonsillar pillars (lower); (B) images of a healthcare worker using the OPAD to take a pharyngeal swab in the isolation wards; during the procedure, the healthcare worker stands to the side of or behind the patients; (C) ROC curves for correlation of the OPAD with the traditional method in collecting swab samples. OPAD, optimized pharyngeal swab assisted device.

patients were documented. According to the Diagnosis and Treatment Protocol for COVID-19 (Trial Version 7), the typical findings of chest CT were bilateral multiple lobular and subsegmental areas of consolidation, bilateral groundglass opacity, and subsegmental areas of consolidation. When compared to the CT results on admission, the results of the most recent chest CT were defined as ameliorated, no significant change, or deteriorated.

\section{Statistical analysis}

Data were analyzed with IBM SPSS V.19 software (Armonk, NY, USA). A descriptive analysis was performed and quantitative data were presented as mean $\pm \mathrm{SD}$ (minimum, maximum) or as median (minimum, maximum), and the counting data were presented as count (percentage of total). The McNemar test and the kappa statistic were conducted to test the significance of the difference between the 


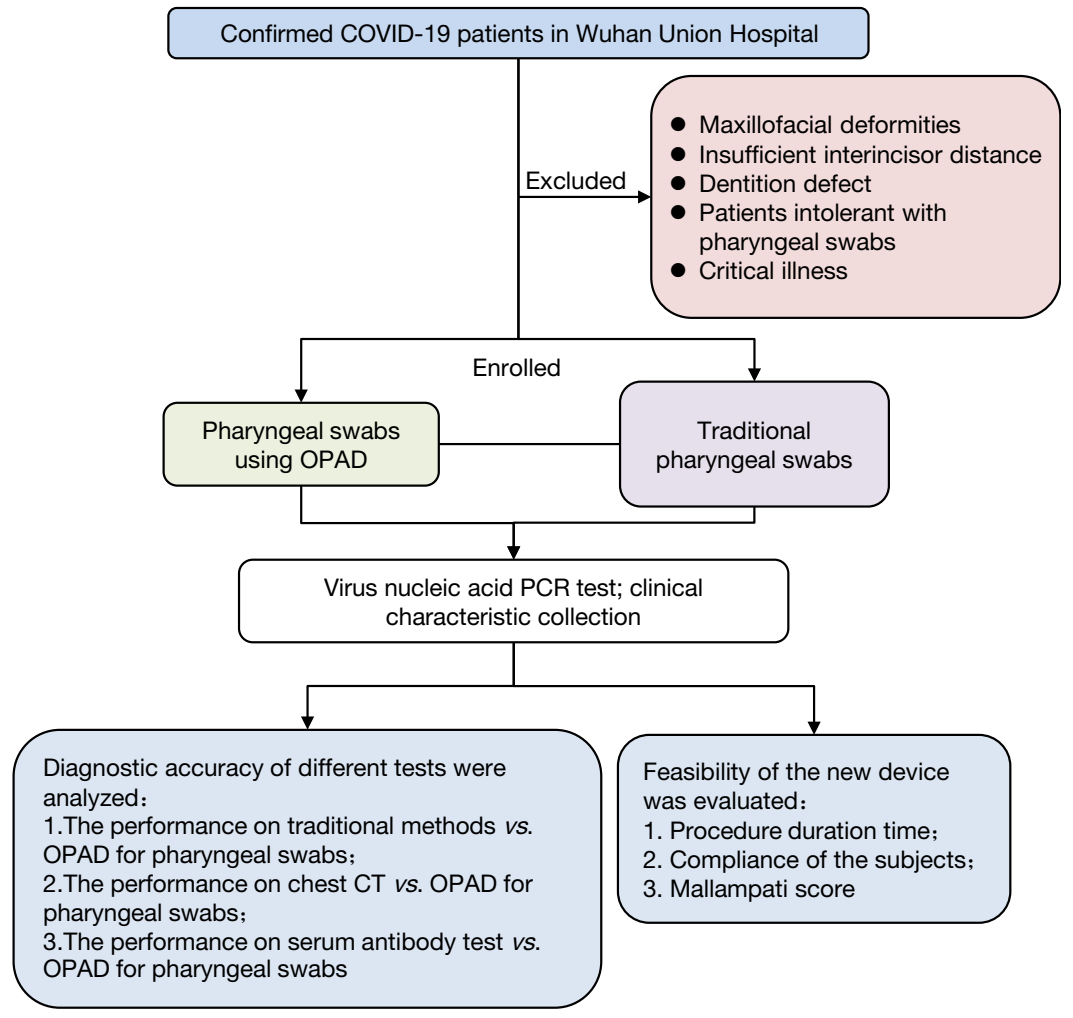

Figure 2 Flowchart of the inclusion and exclusion of patients for the present study. OPAD, optimized pharyngeal swab assisted device; CT, computed tomography.

efficacies of the two diagnostic procedures. A $\mathrm{P}$ value $<0.05$ was regarded as being statistically significant.

\section{Results}

\section{The operator performing pharyngeal swabs did not always} get a satisfactory field of vision

The questionnaire results obtained from 82 healthcare workers from the frontline of designated infection hospitals in China during the SARS-CoV-2 pandemic were analyzed and summarized. Each participant met the inclusion criteria of having had performed the pharyngeal swabs at least once. Among all the respondents, only 5 doctors $(6.1 \%)$ were specialists from the infectious diseases or respiratory department, with the other 77 drawn from other divisions. The pharyngeal swabs had been performed $>5$ times by $41.5 \%$ ( 34 of 82 ) of respondents, and $<10 \%$ ( 8 of 82 ) had never worried about the risk of cross-infection, despite all of them having worn adequate PPE (Figure 3A,B). About 38\% (31 of 82 ) of the operators claimed that they could always achieve the full exposure of the pharyngeal wall of the patient (Figure 3A), with a lack of full exposure potentially compromising the operation of swabbing. The results of the survey supported the hypothesis that insufficient exposure of the pharyngeal wall and fear of viral cross-infection partially limited the accuracy of RT-PCR of pharyngeal sampling.

\section{The OPAD design}

Pharyngeal swab sampling is hazardous as the operator has to be near the patient (10) (Figure 3C), and the procedure itself may generate aerosols and droplets. For the traditional procedure, the operator and patient face each other, and the operator must manually employ a tongue depressor. Pharyngeal swabs may cause the patient to cough or retch, which can obstruct the clear view of the pharyngeal wall. Therefore, we aimed to produce an ameliorative device that could be adapted to oral cavities of different sizes. In doing so, we considered the principles of $3 \mathrm{D}$ printing, including the geometric program and material. The prototype design was a two-part device comprising a display unit and an 
A

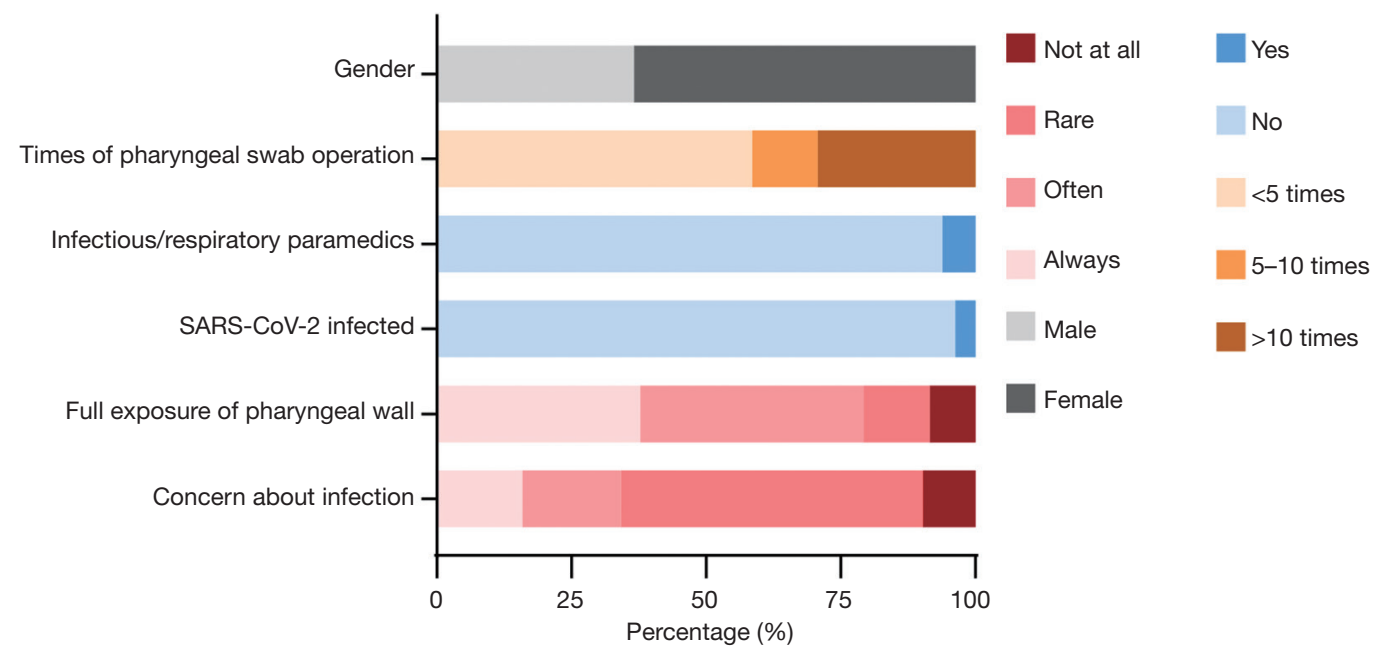

B

PPEs for pharyngeal swabs

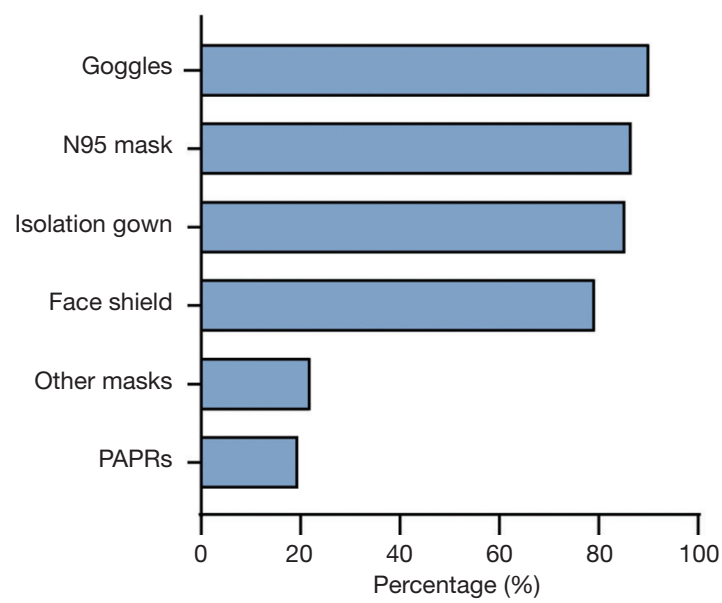

C

Figure 3 Results of the questionnaire for healthcare workers who had performed pharyngeal swabs during the COVID-19 outbreak. (A) The respondent selection frequency from the questionnaire for healthcare workers (the full wording of the questions is found in the supplementary files); (B) the respondents answered "Which PPEs have you used in pharyngeal swab sampling?"; (C) the traditional standard method for sampling a pharyngeal swab-the operator is in close proximity to the patient. PPE, personal protective equipment; PAPR, powered air-purifying respirator.

exposure apparatus, designed to keep the patients' mouth open and function as a self-retaining tongue depressor (Figure 1A). This device fully and steadily exposes the walls of the pharynx to allow ease of sample collection (Figure 1A,B, Videos S1,S2). Referring to the previous reports on the average normal range of mouth opening (11), and the mean value of two-thirds of the tongue length (from the top lip to tip) in 10 volunteers, the height of the oris speculum and tongue spatula were set to 3.5 and $4.5 \mathrm{~cm}$, respectively.
$\mathrm{ABS}$ is a common material used in $3 \mathrm{D}$ printing; it is safe, tractable, tough, and chemical and impact resistant (12). For these reasons, and because of its glossy surface, ABS was chosen for the pharyngeal wall exposure unit of the OPAD. As for the display unit, in addition to a camera with a light source, a data transmission line, and an electronic display screen, a holder was designed so that the light may be adjusted to different angles. The camera could be a laryngoscope, a fiberoptic bronchoscope, or another piece of equipment, such as an industrial endoscope. The design 
Table 1 The clinical characteristics of the subjects

\begin{tabular}{|c|c|}
\hline Characteristics & All patients $(n=22)$ \\
\hline Age $(y)^{*}$ & $63.5[31,79]$ \\
\hline \multicolumn{2}{|l|}{ Gender } \\
\hline Male & $12(54.55)$ \\
\hline Female & $10(45.45)$ \\
\hline \multicolumn{2}{|l|}{ Laboratory investigations } \\
\hline White blood cell count (G/L) & $6.95 \pm 3.16(2.74,17.87)$ \\
\hline Lymphocyte count (G/L) & $1.57 \pm 0.43(0.87,2.52)$ \\
\hline Lymphocyte percentage (\%) & $25.91 \pm 11.48(5.0,44.3)$ \\
\hline C-reactive protein (mg/L) & $9.41 \pm 20.11(0.10,76.76)$ \\
\hline IL-6 (pg/L) & $17.64 \pm 29.0(1.50,132.81)$ \\
\hline \multicolumn{2}{|l|}{ Antibody (13 patients available) } \\
\hline $\lg M(+)$ & $3(23.08)$ \\
\hline $\lg G(+)$ & $12(92.31)$ \\
\hline Procedure duration time (s) & $30 \pm 13[17,78]$ \\
\hline The hospitalized period $(d)^{\star}$ & $36.5[7,47]$ \\
\hline $\begin{array}{l}\text { The interval between the adjacent } \\
\text { pharyngeal swabs }(d)^{\star}\end{array}$ & $2[1,8]$ \\
\hline \multicolumn{2}{|l|}{ The most recent CT scan } \\
\hline Ameliorated & 20 (90.90) \\
\hline No significant change & $1(4.55)$ \\
\hline Deteriorated & $1(4.55)$ \\
\hline \multicolumn{2}{|l|}{ Mallampati score } \\
\hline 1 & $17(77.3)$ \\
\hline 2 & $4(18.2)$ \\
\hline 3 & $1(4.5)$ \\
\hline 4 & $0(0.0)$ \\
\hline
\end{tabular}

Quantitative data were presented as mean \pm SD (minimum, maximum), the counting data were presented as count (percentage of total); ${ }^{*}$, data were presented as median (minimum, maximum).

philosophy for the OPAD was to make it safer and easier to obtain pharyngeal swab samples from patients under various conditions

\section{Efficacy assessment in COVID-19 patients}

To test the accuracy of the device, 23 confirmed COVID-19 patients in Wuhan Union Hospital participated, one of whom withdrew from the cohort due to discomfort during the pharyngeal swabbing (Table 1 and Table S1). There were 12 men $(54.55 \%)$ among the remaining 22 patients. The patients had a median age of 63.5 (range, 31-79) years and a median hospital stay of 36.5 (range, 7-47) days. The median interval time of the two sequential tests of the pharyngeal swab was 2 (range, 1-8) days. All patients had chest CT abnormalities at baseline. The results of laboratory examinations were mildly abnormal, with the most common anomaly being an elevated serum interleukin-6 (IL-6) level $(17.64 \pm 29.0 \mathrm{pg} / \mathrm{L})$. The routine blood tests and C-reactive protein were often normal, while most of the immunoglobulin $M(\operatorname{IgM})(23.08 \%) /$ immunoglobulin G (IgG) $(92.31 \%)$ combined antibody tests for SARS-CoV-2 were positive.

The MS was utilized to assess the visibility of oropharyngeal structures during the pharyngeal swabs, in which a higher the score indicated a higher probability of a challenging swabbing. The pharyngeal structure was exposed well in the majority of patients when the operators used the OPAD. Of the patients, 17 (77.3\%) had an MS of 1 and 4 (18.2\%) had an MS of 2, which meant the operator could acquire the swab samples without difficulty. In all 22 patients, sampled a total of 44 times, we returned 18 pairs $(81.82 \%, 18 / 22)$ of consistent results using the two different methods, and three $(13.64 \%, 3 / 22)$ single OPAD-positive qRT-PCR results in the four contradictions $(18.18 \%, 3 / 22)$ (Table 2). The kappa coefficient between the two methods was $0.639(\mathrm{P}=0.002)$, which meant that the similarities between the two diagnostic methods were significant, albeit moderately. The positive rate was higher using the OPAD $(54.55 \%, 12 / 22)$ than using the traditional method $(45.45 \%$, $10 / 22)$, which suggested that the OPAD could perform better in pharyngeal sampling, despite the McNemar test revealing no statistically significant differences $(\mathrm{P}=0.625)$. The ROC analysis for the sensitivity and specificity of using the OPAD versus the traditional method also indicated that the OPAD showed good efficacy in pharyngeal sampling for COVID-19 patients (Figure 1C, AUC =0.825). There was only one patient that returned a positive result with the traditional swab and a negative result with OPAD. A test after 2 days indicated that this patient also returned a negative result with the traditional swab. Interestingly, when comparing the results of chest CT and qRT-PCR obtained using OPAD, both of the patients with no amelioration on CT had a positive qRT-PCR result with OPAD, while only one of them was positive with the traditional sampling 
Table 2 Comparison of qRT-PCR results between OPAD and traditional methods in pharyngeal swabs

\begin{tabular}{lccc}
\hline $\begin{array}{l}\text { Traditional method for } \\
\text { pharyngeal swabs }\end{array}$ & Positive & OPAD & Total \\
\cline { 2 - 3 } & $9(40.91)$ & $1(4.55)$ & $10(45.45)$ \\
Positive & $3(13.64)$ & $9(40.91)$ & $12(54.55)$ \\
Negative & $12(54.55)$ & $10(45.45)$ & 22 \\
Total & & Negative & \\
\hline
\end{tabular}

Data were presented as count (percentage of total). OPAD, optimized pharyngeal swab assisted device.

Table 3 Efficacy of OPAD versus traditional method for swab sampling

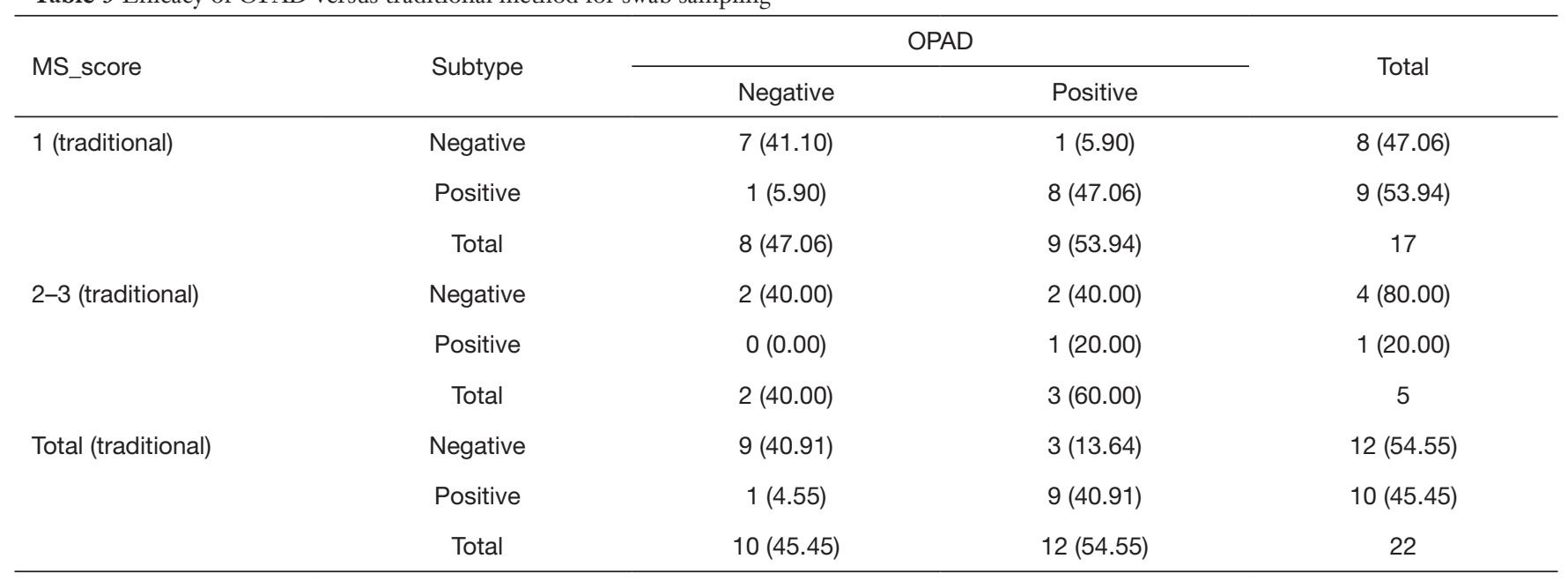

Data were presented as count (percentage of total). OPAD, optimized pharyngeal swab assisted device.

(Table S2). Moreover, the efficacy of OPAD was evaluated in a subgroup analysis based on MS. Of the five patients with higher MS (MS $=2-3$ ), four $(80 \%)$ had a positive diagnosis using OPAD, while only one (20\%) was positive using the traditional method (Table 3). Tolerance assessment was based on an evaluation of patient compliance and the procedure duration time with OPAD. There was no unacceptable discomfort reported by the patients, and the average procedure duration of sampling was $22 \mathrm{~s}(22 \pm 11 \mathrm{~s})$.

\section{Discussion}

During the outbreak of SARS in 2003, the infection rate of healthcare workers was relatively high (13), especially among healthcare workers who performed aerosolproducing procedures, such as endotracheal intubation. Sampling with the pharyngeal swab is also risky, as the operator is in close direct contact with the patient, and the face-to-face procedure can easily cause respiratory droplet transmission. The large volume and surge in demand for such a test to be performed also amplified the risk of exposure for the operator. The operator of the pharyngeal swab is required to use a tongue depressor to hold the patient's tongue out of the way and perform a sweeping motion to swab the pharyngeal wall and tonsillar pillars (10). The Chinese CDC recommends that healthcare staff use enhanced droplet/airborne PPE, incorporating the use of a powered air-purifying respirator (PAPR) when performing pharyngeal swabs of patients who potentially have SARSCoV-2. However, due to a global PPE shortage, surgical masks and surgical respirators are the only options in many primary hospitals. According to our questionnaire, healthcare workers also have doubts regarding protective efficacy and fear of the risk of cross-infection.

In this study, we proposed an economical medical device allowing healthcare workers to simply perform the swab standing at the side of or behind the patients, and reasonably inferred that it could reduce the probability of nosocomial cross-transmission. Without a complex structure, each component of the OPAD was framed and 
manufactured considering the manufacturer's accessibility and the acceptance of healthcare workers and patients, along with clinical safety. Given the rapid pace of SARS-CoV-2 infection worldwide, the implementation of our upgraded and economical device (costing about $\$ 6$ for the pharynx exposure unit) for pharyngeal swabbing is urgently needed for the benefit of public health. Most developing countries, and even some developed countries, lack adequate PPE, such as gowns, surgical masks, N95 masks, and PAPR; our device partly compensates for the insufficiency of personal protection.

Although the SARS-CoV-2 IgG-IgM combined antibody test is currently available in clinical practice (14), the pharyngeal swab is still an indispensable procedure for etiological diagnosis of COVID-19. It reflects the presence and activity of the virus and must be carried out repeatedly (at least five times) throughout the hospitalization of every patient. Therefore, the popularization of our OPAD could reduce the chance of infection and safeguard healthcare workers, not only in China but also worldwide in the ongoing pandemic situation.

Furthermore, the positivity rate of pharyngeal swabs remains relatively low $(38-78 \%)(15,16)$, and it has been reported that "cured" patients can still return positive results from RT-PCR tests on pharyngeal swabs, even up to 13 days after discharge (17). We speculated that this was partly due to incorrect operation on the behalf of the pharyngeal swab operator. To evaluate the degree of pharyngeal wall exposure more precisely, we employed the MS, one of the clinical tests most widely used by anesthetists during preoperative physical examinations. When sampled using the OPAD, the pharyngeal wall and bilateral tonsils can be well exposed using the combination of an auxiliary lighting system and tongue spatula. The OPAD keeps the patient's mouth open and tongue pressed down simultaneously, which means the healthcare worker can avoid close exposure and be less anxious while performing the procedure. This may also improve the accuracy of swab sampling, especially for patients with higher MS scores, and has great significance for the diagnosis and recovery assessment of COVID-19 patients.

A limitation of our study was the relatively small number of participants included in the efficacy test. There are currently very few newly confirmed COVID-19 cases in Wuhan, with most patients in a state of recovery, as inferred by their clinical characteristics. Another drawback is that most of the patients (77\%) enrolled in this study had an MS of 1 which indicates a selection bias; however, we believe that the device and ideas we have created can be tested and further validated by medical institutions in other epidemic areas. Although our OPAD did not have statistically significant superiority in this clinical study using a small sample size, the results suggested that the advantages of the OPAD include easy accessibility, high efficiency, and cost-effectiveness. This optimized device allows healthcare workers to avoid having to perform the pharyngeal swab at close range, without the need to undertake extra professional training. As the COVID-19 pandemic escalates, and the potential for future outbreaks grows, the OPAD can be utilized efficiently and to safeguard healthcare workers worldwide.

\section{Acknowledgments}

We would like to thank the members of Beijing Smart Technology Co., Ltd. for their excellent technical assistance. Funding: None.

\section{Footnote}

Reporting Checklist: The authors have completed the MDAR reporting checklist. Available at http://dx.doi.org/10.21037/ atm-20-3612

Data Sharing Statement: Available at http://dx.doi. org/10.21037/atm-20-3612

Peer Review File: Available at http://dx.doi.org/10.21037/ atm-20-3612

Conflicts of Interest: All authors have completed the ICMJE uniform disclosure form (available at http://dx.doi. org/10.21037/atm-20-3612). Dr. JJ, Dr. HTT and Dr. JT have a patent "A medical tooth retractor and auxiliary equipment for pharyngeal exposure observation" licensed to ZL 20202 0303125.2. The other authors have no conflicts of interest to declare.

Ethical Statement: The authors are accountable for all aspects of the work in ensuring that questions related to the accuracy or integrity of any part of the work are appropriately investigated and resolved. This clinical study was conducted in accordance with the principles of the Declaration of Helsinki (as revised in 2013) from the International Conference on Harmonization. All patients gave written informed consent to participate in this study. 
The study received ethical approval from the Tongji Medical College Ethics Committee.

Open Access Statement: This is an Open Access article distributed in accordance with the Creative Commons Attribution-NonCommercial-NoDerivs 4.0 International License (CC BY-NC-ND 4.0), which permits the noncommercial replication and distribution of the article with the strict proviso that no changes or edits are made and the original work is properly cited (including links to both the formal publication through the relevant DOI and the license). See: https://creativecommons.org/licenses/by-nc-nd/4.0/.

\section{References}

1. Huang C, Wang Y, Li X, et al. Clinical features of patients infected with 2019 novel coronavirus in Wuhan, China.

Lancet 2020;395:497-506.

2. Wu Z, McGoogan JM. Characteristics of and Important Lessons From the Coronavirus Disease 2019 (COVID-19) Outbreak in China: Summary of a Report of 72314 Cases From the Chinese Center for Disease Control and Prevention. JAMA 2020;323:1239-42.

3. Kluytmans-van den Bergh MFQ, Buiting AGM, Pas SD, et al. Prevalence and Clinical Presentation of Health Care Workers With Symptoms of Coronavirus Disease 2019 in 2 Dutch Hospitals During an Early Phase of the Pandemic. JAMA Netw Open 2020;3:e209673.

4. Remuzzi A, Remuzzi G. COVID-19 and Italy: what next? Lancet 2020;395:1225-8.

5. Li L, Cheng S, Gu J. SARS infection among health care workers in Beijing, China. JAMA 2003;290:2662-3.

6. Guan WJ, Ni ZY, Hu Y, et al. Clinical Characteristics of Coronavirus Disease 2019 in China. N Engl J Med 2020;382:1708-20.

7. Mallampati SR, Gatt SP, Gugino LD, et al. A clinical sign to predict difficult tracheal intubation: a prospective study.

Cite this article as: Jia J, Fang ST, Feng XB, Tong W, Tian HT, Tang J. Application of an optimized pharyngeal swab-assisted device in pharyngeal sampling for COVID-19 patients. Ann Transl Med 2021;9(4):319. doi: 10.21037/atm-20-3612
Can Anaesth Soc J 1985;32:429-34.

8. Detsky ME, Jivraj N, Adhikari NK, et al. Will This Patient Be Difficult to Intubate?: The Rational Clinical Examination Systematic Review. JAMA 2019;321:493-503.

9. Metlay JP, Waterer GW, Long AC, et al. Diagnosis and Treatment of Adults with Community-acquired Pneumonia. An Official Clinical Practice Guideline of the American Thoracic Society and Infectious Diseases Society of America. Am J Respir Crit Care Med 2019;200:e45-e67.

10. Organization WH. Collecting, preserving and shipping specimens for the diagnosis of avian influenza A (H5N1) virus infection: guide for field operations: World Health Organization; 2006.

11. Mezitis M, Rallis G, Zachariades N. The normal range of mouth opening. J Oral Maxillofac Surg 1989;47:1028-9.

12. Zhang H, Cai L, Golub M, et al. Tensile, creep, and fatigue behaviors of 3D-printed acrylonitrile butadiene styrene. J Mater Eng Perform 2018;27:57-62.

13. Twu SJ, Chen TJ, Chen CJ, et al. Control measures for severe acute respiratory syndrome (SARS) in Taiwan. Emerg Infect Dis 2003;9:718-20.

14. Li Z, Yi Y, Luo X, et al. Development and Clinical Application of A Rapid IgM-IgG Combined Antibody Test for SARS-CoV-2 Infection Diagnosis. J Med Virol 2020;92:1518-24.

15. Ren XS, Liu Y, Chen HT, et al. Application and optimization of RT-PCR in diagnosis of SARS-CoV-2 infection. MedRxiv 2020. doi: https://doi.org/10.1101/202 0.02.25.20027755.

16. Liu R, Han H, Liu F, et al. Positive rate of RT-PCR detection of SARS-CoV-2 infection in 4880 cases from one hospital in Wuhan, China, from Jan to Feb 2020. Clin Chim Acta 2020;505:172-5.

17. Lan L, Xu D, Ye G, et al. Positive RT-PCR Test Results in Patients Recovered From COVID-19. JAMA 2020;323:1502-3. 


\section{Supplementary}

\section{Appendix 1 The Questionnaire for the medical staff who has ever performed the pharyngeal swab during COVID-19 pandemic}

1. What is your gender?
A. Male
B. Female

2. What is your age?

3. Are you a specialist from infection or pneumology department, or ICU?
A. Yes
B. No

4. How many times have you performed the pharyngeal swab during the pandemic of COVID-19?
A. Less than 5 times
B. Between 5 to 10 times
C. More than 10 times

5. Do you have fear for the risk of being infected of SARS-CoV-2 during pharyngeal swabs?
A. Not at all
B. rare
C. Often
D. Always

6. During the operation of pharyngeal swabs, did you fully exposed the bilateral tonsil and pharyngeal and got a clear operative field?
A. Not at all
B. rare
C. Often
D. Always

7. Have you been infected by the SARS-CoV-2?
A. Yes
B. No

8. Which personal protective equipment (PPE) have you wore when you proceeded the pharyngeal swabs? (multiple-choice question)
A. Goggles;
B. N95 masks;
C. other medical mask;
D. Isolation gown;
E. Face shield;
F. Powered air purifying respirator (PAPR)

9. The location of the medical institution you served in the COVID-19 pandemic:
A. Wuhan, Hubei Province, China;
B. Hubei Province except Wuhan, China
C. Province other than Hubei;
D. Countries other than China 
Table S1 The detailed clinical data of the subject

\begin{tabular}{|c|c|c|c|c|c|c|c|c|c|c|c|c|c|c|c|c|c|c|c|c|c|c|c|}
\hline No. & Gender & Age & Intervals & $\begin{array}{l}\text { Traditional } \\
\text { date }\end{array}$ & Traditional & OPAD date & OPAD & $\begin{array}{l}\text { Procedure } \\
\text { duration (s) }\end{array}$ & MS Score & BR date & $\begin{array}{l}\text { White blood } \\
\text { cell count (G/L) }\end{array}$ & $\begin{array}{l}\text { Lymphocyte } \\
\text { count (G/L) }\end{array}$ & $\begin{array}{l}\text { Lymphocyte } \\
\text { percentage (\%) }\end{array}$ & CRP date & $\begin{array}{l}\text { CRP (mg/ } \\
\text { L) }\end{array}$ & IL-6 date & IL-6 (pg/L) & $\begin{array}{l}\text { Antibody } \\
\text { date }\end{array}$ & $\lg M$ & $\lg G$ & Last chest CT & $\begin{array}{l}\text { Admission } \\
\text { date }\end{array}$ & $\begin{array}{l}\text { hospitalization } \\
\text { time }\end{array}$ \\
\hline 1 & Female & 50 & 2 & 2020/3/19 & - & 2020/3/21 & - & 15 & 1 & 2020/3/20 & 7.86 & 1.71 & 21.7 & 2020/3/16 & 0.70 & 2020/3/15 & 1.50 & NA & NA & NA & ameliorated & 2020/2/3 & 47 \\
\hline 2 & Female & 48 & 3 & 2020/3/16 & - & 2020/3/19 & - & 17 & 1 & 2020/3/13 & 4.84 & 1.49 & 30.9 & 2020/3/13 & 1.23 & 2020/3/15 & 6.96 & 2020/3/20 & - & + & ameliorated & 2020/3/12 & 7 \\
\hline 3 & Male & 72 & 1 & 2020/3/17 & - & 2020/3/18 & + & 25 & 3 & 2020/3/18 & 8.49 & 0.87 & 10.3 & 2020/3/18 & 1.05 & NA & NA & NA & NA & NA & ameliorated & 2020/2/14 & 33 \\
\hline 4 & Male & 38 & 2 & 2020/3/16 & - & 2020/3/18 & - & 65 & 1 & 2020/3/16 & 7.45 & 1.58 & 21.2 & 2020/3/16 & 1.81 & 2020/3/15 & 3.00 & $2020 / 3 / 20$ & - & + & ameliorated & 2020/2/20 & 27 \\
\hline 5 & Male & 73 & 2 & 2020/3/13 & + & 2020/3/15 & + & 19 & 1 & 2020/3/20 & 6.80 & 0.87 & 12.8 & 2020/3/20 & 1.07 & 2020/3/20 & 4.90 & $2020 / 3 / 20$ & + & + & ameliorated & 2020/2/15 & 29 \\
\hline 6 & Female & 67 & 3 & 2020/3/16 & - & 2020/3/19 & - & 20 & 2 & 2020/3/19 & 4.29 & 1.89 & 43.9 & 2020/3/19 & 11.39 & 2020/3/16 & 30.91 & 2020/3/16 & - & + & ameliorated & 2020/2/7 & 41 \\
\hline 7 & Male & 69 & 4 & 2020/3/11 & + & 2020/3/15 & + & 22 & 1 & $2020 / 3 / 20$ & 3.23 & 1.91 & 36.6 & NA & NA & 2020/3/11 & 6.63 & NA & NA & NA & ameliorated & 2020/2/8 & 36 \\
\hline 8 & Male & 61 & 1 & 2020/3/18 & + & 2020/3/19 & - & 19 & 1 & 2020/3/18 & 7.63 & 1.66 & 21.7 & 2020/3/18 & 5.81 & 2020/3/16 & 23.36 & 2020/3/18 & + & + & ameliorated & 2020/2/6 & 42 \\
\hline 9 & Male & 55 & 2 & 2020/3/14 & + & 2020/3/16 & + & 18 & 2 & 2020/3/16 & 6.60 & 1.97 & 32.5 & 2020/3/16 & 1.37 & 2020/3/16 & 1.50 & 2020/3/14 & - & + & ameliorated & 2020/2/5 & 40 \\
\hline 10 & Male & 44 & 2 & 2020/3/13 & + & 2020/3/15 & + & 9 & 1 & 2020/3/14 & 8.97 & 1.64 & 32 & 2020/3/14 & 3.64 & 2020/3/12 & 1.50 & NA & NA & NA & ameliorated & 2020/2/7 & 37 \\
\hline 11 & Female & 64 & 8 & 2020/3/11 & + & 2020/3/19 & + & 18 & 1 & 2020/3/19 & 8.20 & 2.52 & 40.6 & 2020/3/5 & 0.10 & 2020/3/19 & 5.96 & 2020/3/15 & - & + & ameliorated & $2020 / 2 / 10$ & 38 \\
\hline 12 & Male & 65 & 5 & 2020/3/14 & - & 2020/3/19 & - & 15 & 1 & 2020/3/20 & 5.74 & 1.43 & 24.8 & 2020/3/20 & 2.09 & 2020/3/17 & 5.16 & NA & NA & NA & ameliorated & 2020/2/1 & 47 \\
\hline 13 & Female & 62 & 1 & 2020/3/15 & - & 2020/3/16 & - & 17 & 1 & 2020/3/16 & 5.33 & 1.46 & 27.3 & 2020/3/9 & 64.25 & 2020/3/16 & 9.30 & 2020/3/16 & - & + & ameliorated & 2020/2/3 & 42 \\
\hline 14 & Female & 32 & 2 & 2020/3/18 & + & 2020/3/20 & + & 24 & 1 & 2020/3/15 & 10.51 & 1.40 & 13.3 & NA & NA & 2020/3/19 & 4.70 & 2020/3/20 & - & + & ameliorated & 2020/2/19 & 30 \\
\hline 15 & Female & 31 & 3 & 2020/3/14 & + & 2020/3/17 & + & 20 & 1 & 2020/3/14 & 4.82 & 2.03 & 42.1 & NA & NA & 2020/3/18 & 4.58 & 2020/3/10 & - & + & ameliorated & 2020/2/16 & 30 \\
\hline 16 & Female & 79 & 1 & 2020/3/15 & - & 2020/3/16 & + & 26 & 2 & 2020/3/18 & 17.87 & 0.89 & 5 & 2020/3/16 & 76.76 & 2020/3/18 & 132.81 & 2020/3/18 & - & - & $\begin{array}{l}\text { no significant } \\
\text { change }\end{array}$ & $2020 / 3 / 2$ & 14 \\
\hline 19 & Female & 68 & 2 & 2020/3/17 & - & 2020/3/19 & - & 27 & 1 & 2020/3/17 & 4.90 & 1.25 & 25.5 & NA & NA & 2020/3/17 & 1.86 & NA & NA & NA & ameliorated & 2020/2/9 & 39 \\
\hline 20 & Male & 55 & 2 & 2020/3/15 & - & 2020/3/17 & + & 19 & 1 & 2020/3/12 & 5.88 & 1.59 & 27.4 & 2020/3/16 & 8.54 & 2020/3/16 & 9.46 & NA & NA & NA & ameliorated & 2020/2/9 & 37 \\
\hline 21 & Male & 78 & 2 & 2020/3/13 & + & 2020/3/15 & + & 24 & 1 & 2020/3/17 & 2.74 & 0.96 & 11.3 & 2020/3/17 & 1.59 & 2020/3/13 & 43.61 & NA & NA & NA & deteriorated & 2020/2/9 & 35 \\
\hline 22 & Male & 63 & 3 & 2020/3/12 & + & 2020/3/15 & + & 15 & 1 & 2020/3/13 & 9.46 & 1.66 & 15.4 & 2020/3/17 & 6.13 & 2020/3/17 & 7.53 & NA & NA & NA & ameliorated & 2020/2/9 & 35 \\
\hline
\end{tabular}


Table S2 Comparison of qRT-PCR results for OPAD in pharyngeal swabs and chest CT

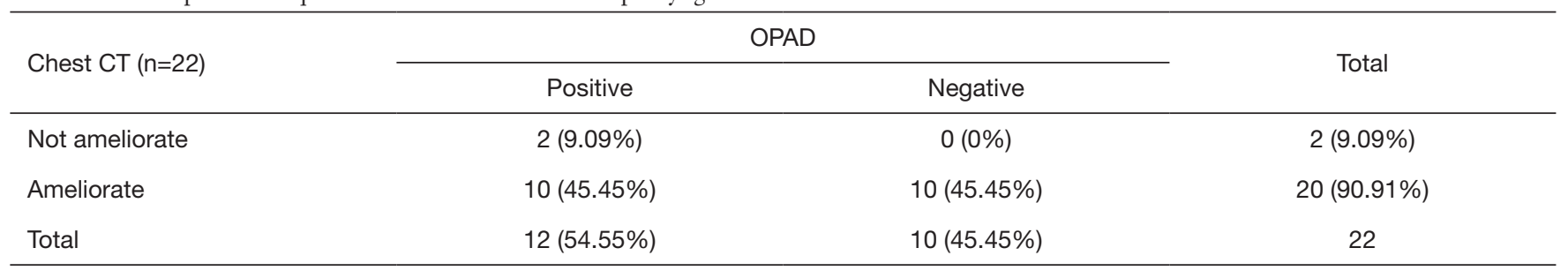

